\title{
Ultrasound Imaging of Early Extraembryonic Structures
}

\author{
${ }^{1}$ Sándor Nagy, ${ }^{2}$ Zoltán Papp
}

\begin{abstract}
Transvaginal sonography is the most useful diagnostic method to visualize the early pregnancy, to determine whether it is intrauterine or extrauterine (ectopic), viable or not. Detailed examination of extraembryonic structures allows us to differentiate the types of early pregnancy failures and highlights the backgrounds of vaginal bleeding, as the most frequent symptom of the first trimester of gestation. The reliable ultrasonographic sign of an intrauterine pregnancy is visualization of double decidual ring, which represents the trophoblast's layer. The abnormality in the sonographic appearance of a gestational sac, a yolk sac, and a chorionic plate can predict subsequent embryonic damage and death.
\end{abstract}

Keywords: Blighted ovum, Chorionic plate, Extraembryonic structures, Gestational sac, Missed abortion, Subchorionic hemorrhage, Yolk sac.

How to cite this article: Nagy S, Papp Z. Ultrasound Imaging of Early Extraembryonic Structures. Donald School J Ultrasound Obstet Gynecol 2017;11(1):11-19.

Source of support: Nil

Conflict of interest: None

\section{INTRODUCTION}

The use of ultrasonography in the very early stages of pregnancy enables to confirm the status of intrauterine living embryo or diagnose extrauterine pregnancy for which medical treatment with low morbidity is feasible with early detection. First trimester ultrasonography before 12-weeks of gestation is a valuable tool for predicting pregnancy outcomes, particularly with respect to detecting complicated or unviable pregnancies with the exception of aneuploidy. ${ }^{1}$ Transvaginal sonography of first trimester pregnancy is a core bedside ultrasound application, an essential component of the evaluation of pregnant women, and helps to vizualize the early embryonic and extraembryonic structures. Along with other clinical data, ultrasound findings allow obstetricians

\footnotetext{
${ }^{1}$ Associate Professor, ${ }^{2}$ Honorary Professor

${ }^{1}$ Department of Obstetrics and Gynecology, Aladár Petz County Teaching Hospital, Győr, Hungary

${ }^{2}$ Maternity Private Hospital for Obstetrics and Gynecology Semmelweis University, Budapest, Hungary
}

Corresponding Author: Zoltán Papp, Honorary Professor Maternity Private Hospital for Obstetrics and Gynecology Semmelweis University, Budapest, Hungary, e-mail: papp. zoltan@maternity.hu to arrive at an accurate diagnosis and appropriate disposition, thus providing efficient care that benefits both patients and doctors. The specific sonographic appearance of normal pregnancy depends upon the gestational age. As the gestational age increases, the ability to assess the location and normal development of the pregnancy becomes better.

Spontaneous abortion is one of the most common complications of pregnancy; every 12 to 15 out of 100 conceptus are miscarried in the first half of gestation. Vaginal bleeding is one of the most serious symptoms of the spontaneous abortion, which the pregnant are afraid of, especially when extrachorial bleeding is detected by ultrasound.

Transvaginal sonography is the optimal way to image a patient with developing gestation. Indications for this examination include not only the need to identify the location and number of gestational sacs (GSs), date the age of a pregnancy, and determine whether the embryo has a normal appearance, but also to vizualize the extraembryonic structures, which help to predict early pregnancy failures. The first visible structures in pregnancy are extraembryonic parts of the gestation. In order to predict the outcome of pregnancy, we need to fully appreciate the development and pathological conditions of early extraembryonic structures.

\section{GESTATIONAL SAC}

The GS corresponds to the chorionic cavity. Its size has been used to evaluate normal progress of the early pregnancy. After 7 days of conception, the blastocyst reaches the uterine cavity. It embeds into the secretory phase endometrium, and begins to implant. The trophoblast cells penetrate into the decidualized endometrium, erode the maternal capillaries, and make contact with the maternal circulation. The trophoblast and the decidual cells form the GS. It is the first visible structure in early pregnancy. It can be detected by transvaginal ultrasonography after the 4 th menstrual week.

The GS is a large cavity of fluid surrounding the embryo. During early embryogenesis, it consists of the extraembryonic coelom, also called the chorionic cavity. By approximately 9 weeks of gestational age, the amniotic sac has expanded to occupy the majority of its volume, eventually filling the entire space. The GS is usually contained with the uterus. It is the only available structure 
that can be used to determine if an intrauterine pregnancy (IUP) exists until the embryo is identified. The GS is an anechoic fluid-filled collection surrounded by a thickened decidua within the uterus that represents an early IUP. The appearance of a GS is the earliest sign of an IUP. Using vaginal transducers with frequencies of at least $5 \mathrm{MHz}$, the earliest evidence of a GS is seen at 4 to 5 weeks and appears as a small 2 to $3 \mathrm{~mm}$ cystic structure embedded in thickened endometrium; this appearance has been described as the intradecidual sign. Small sacs are usually round, but with growth they frequently become elliptic.

The GS is a ring-shaped structure with a doublelayered wall, embedded eccentrically into the endometrium. It is important to differentiate it from the pseudogestational sac of ectopic pregnancy (Table 1). The pseudogestational sac is in fact a fluid-filled cavity between the layers of the enlarged endometrium. It has a thin simple wall, and is usually in central position. Peritrophoblastic activity is never visible in the underlying layers (Figs $1 \mathrm{~A}$ and B). Approximately $20 \%$ of ectopic pregnancies have an intrauterine pseudogestational sac and it is important to be aware of the rare possibility of concurrent intrauterine and extrauterine pregnancy. More recent studies have found a somewhat lower incidence of approximately $10 \%$. It is likely that many of the early descriptions of pseudogestational sacs were due to hypoechoic areas in the decidua appearing anechoic, since we have rarely seen pseudogestational sacs in recent years. $^{2}$

Table 1: The difference between gestational and the pseudogestational sac

\begin{tabular}{lll}
\hline & Gestational sac & Pseudogestational sac \\
\hline Localization & Lateral & Central \\
Shape & Spheroid & Ovoid \\
Contour & Thick, double- & Thin, mono-layered \\
& layered wall & wall \\
$\begin{array}{l}\text { Peritrophoblastic } \\
\text { circulation }\end{array}$ & Exists & Nonexistent \\
\hline
\end{tabular}
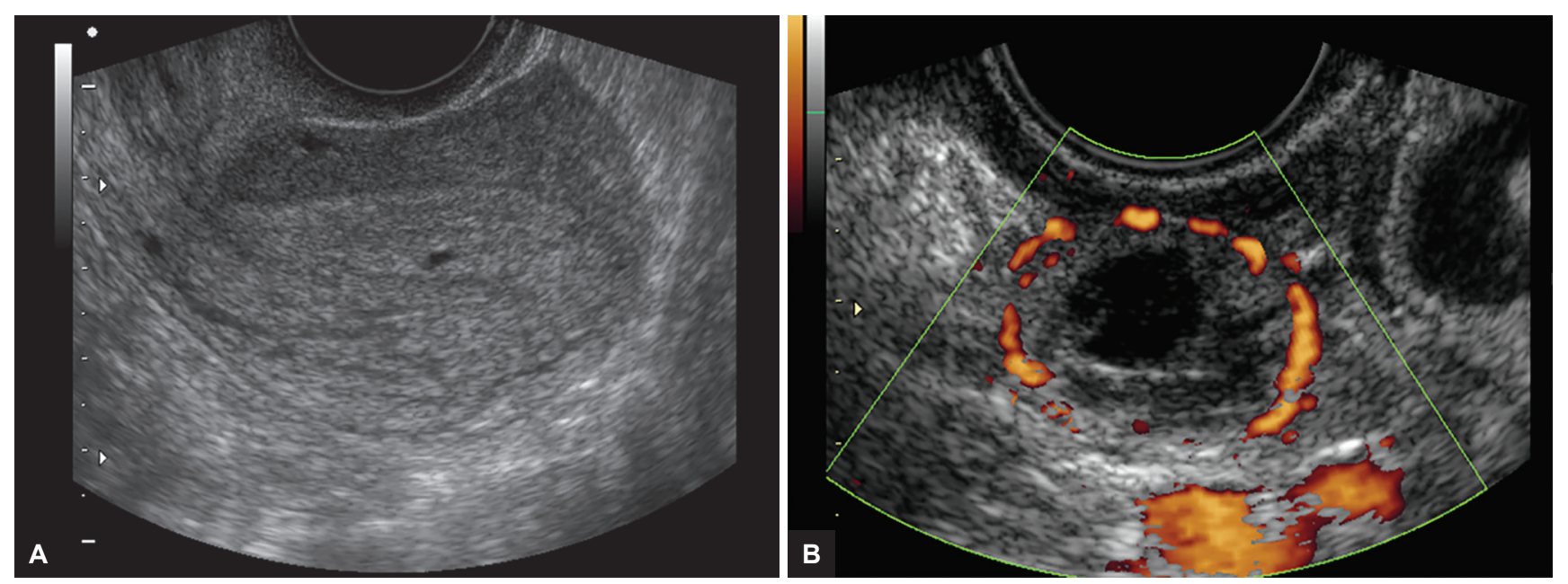

Figs 1A and B: Pseudogestational sac (A) Dilated tuba (B) ectopic pregnancy
The size of the GS also correlates with the health of the developing fetus. If a GS is small for gestational age, it carries a poor prognosis and a serial sonographic examination should be performed to assess the rate of growth. A GS with a growth rate of $<1 \mathrm{~mm} /$ day usually has a poor prognosis. A small GS may be due to oligohydramnios, which is a predictor of poor outcome, even in the presence of normal embryonic cardiac activity. As a rule of thumb, if the difference between the mean sac diameter (MSD) and crown-rump length are $<5 \mathrm{~mm}$, there is an $80 \%$ risk of pregnancy loss. Using transvaginal sonography, a GS with an MSD of $8 \mathrm{~mm}$ without a yolk sac, and a GS with an MSD > $16 \mathrm{~mm}$ without an embryo are important predictors of a nonviable gestation. A blighted ovum or anembryonic pregnancy is an early failure of the embryo to develop within the GS. A pseudogestational sac is seen with an ectopic pregnancy and corresponds to intrauterine fluid collection rimmed by the endometrium. It is centrally located in the uterine cavity (a true sac is eccentric), has sharp angulations, and has an absent double decidual sign without a yolk sac. The normal GS has a regular contour, whereas an abnormal sac has an irregular contour. Other findings that suggest an abnormal sac include a decidual reaction $<2 \mathrm{~mm}$, low position, absent double decidual sac, decreased echogenecity of choriodecidual reaction, and a small sac. ${ }^{3}$

\section{BLIGHTED OVUM}

A blighted ovum (also known as anembryonic gestation) is a pregnancy in which the very early pregnancy appears normal on an ultrasound scan, but as the pregnancy progresses a visible embryo never develops or develops and is reabsorbed. The presentation of women with an "empty sac" is common, with up to $10 \%$ of patients with uncertain viability in pregnancy. Early normal pregnancies always show a GS, but no detectable embryo during a brief, but finite stage of early development. Once a GS 
has been documented subsequent loss of viability remain around $11 \%$, there is no difference between GS diameter when compared with pregnancy outcome. If an embryo has developed up to $5 \mathrm{~mm}$ length, loss of viability occurs in $7.2 \%$ of cases. Persistence of yolk sac has been found inside the GS after embryonic demise. If the volume of the sac is less than $2.5 \mathrm{~mL}$ and is not increasing in size by at least $75 \%$ over a period of 1 week, the definition of this pathological condition in early pregnancy is a blighted ovum. ${ }^{4}$

The terms blighted ovum and anembryonic pregnancy imply that developmental arrest occurred either before formation of the embryo, or before it is detectable. Despite anembryonic state, trophoblastic tissue often continues to function, resulting in continued GS growth. Trophoblastic appearance described with abnormal choriodecidual or trophoblastic reaction consists of a distorted sac shape, thin $(<2 \mathrm{~mm})$ or irregular "double layer" of choriodecidual tissue.

The diagnosis is based on the absence of embryonic echoes within the GS, large enough for such structures to be visualized (Fig. 2). With ultrasound examination, the MSD is the most useful criterion for determining nonviability. If the MSD is higher than $17 \mathrm{~mm}$ that lacks an embryo or more than $13 \mathrm{~mm}$ without visible yolk sac, the probability for nonviable gestation is about $100 \%$. Deformed shape, low position, and thin decidual reaction are strong indicators of nonviable gestations, but are not $100 \%$ accurate. ${ }^{5}$ When no accurate distinction between viable and nonviable gestations can be made at a single examination, serial examination should be carried out before any active management is advocated.

\section{CHORIONIC PLATE}

By the 8th day of development, the blastocyst is partially embedded in the endometrial stroma. In the area over the embryoblast, the trophoblast differentiates into two

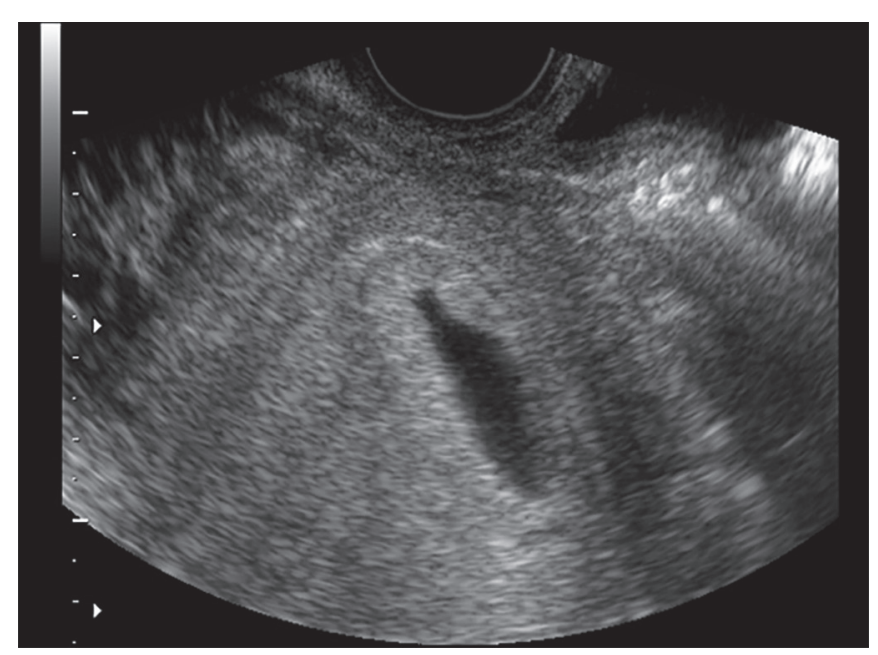

Fig. 2: Blighted ovum in 7-weeks pregnancy layers: (i) An inner layer, the cytotrophoblast, and (ii) an outer zone, the syncytiotrophoblast. The syncytiotrophoblasts penetrate into the stroma and erode the endothelial lining of maternal capillaries. These congested and dilated capillaries are known as sinusoids. The sincytial lacunae then become continuous with the sinusoids, and maternal blood enters the lacunar system. As the trophoblast continues to erode more sinusoids, maternal blood begins to flow through trophoblastic system, thus establishing the uteroplacental circulation. Regular placentation supposes a progressive trophoblast invasion of spiral arteries. Normally, this process is finished by the 20th week. In the majority of abortions and in preeclampsia, this process is disturbed. The evolving chorionic plate becomes thin and fragile, and the trophoblast cork, which hinders the maternal flow in first trimester, is missing. Usually, there is no significant maternal flow in the intervillous space until the 12th week. If the maternal blood floods into the intervillous space in advance, it increases the pressure and mechanically injures and lifts the thin chorionic plate, which leads to abortion (Fig. 3). In fact, regardless of its etiology, this process is in the background of the majority of first trimester abortions.

\section{SUBCHORIONIC HEMORRHAGE}

Subchorionic hemorrhage is defined as bleeding resulting in marginal abruption with separation of the chorion from the endometrial lining. The clinical significance of intrauterine hematoma is controversial. Prior studies have found association between the presence of the hematoma and spontaneous abortion as well as adverse perinatal outcome. There is a great academic and clinical interest in the events of implantation as it is increasingly accepted that these events may determine the future course of gestation. The ultrasound diagnosis can evaluate the morphologic and also the functional parameter of these abnormalities.

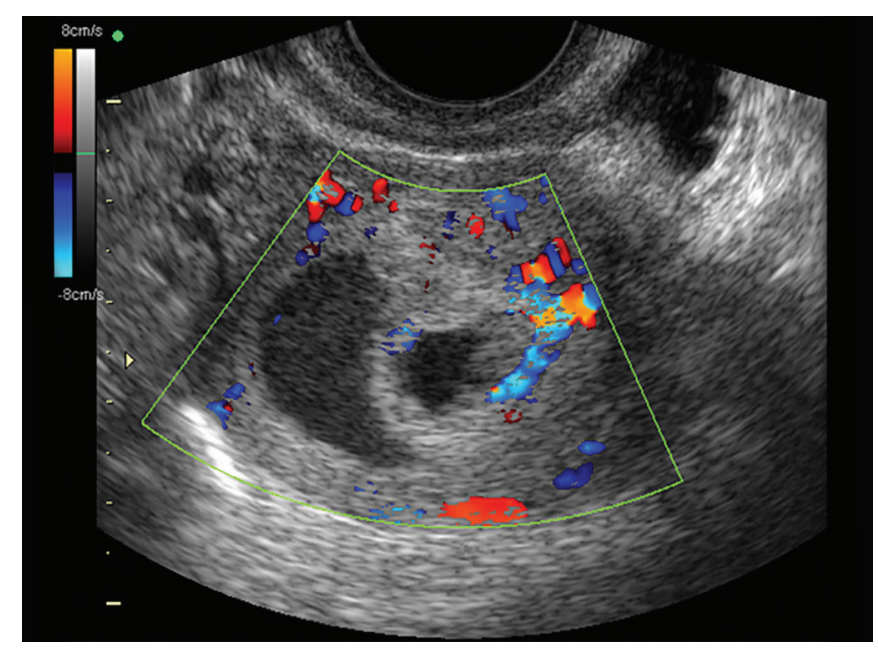

Fig. 3: Subchorionic hemorrhage in 6-weeks pregnancy 
Vaginal bleeding is a frequent complication of pregnancy during the first trimester, with an incidence of 16 to $25 \%$. Intrauterine bleeding without cervical dilatation and tenderness during the early pregnancy period is defined as threatened abortion. Generally, it is not associated with pain and excessive bleeding. These bleedings result in maternal anxiety and may be associated with fetal/ maternal adverse outcomes. The risk of a subplacental hemorrhage appears to be related to gestational age, size, and location of the bleed. One of the suggested mechanisms for threatened abortion is placental dysfunction, which can also cause several late complications, such as preeclampsia, preterm labor, preterm birth, placental abruption, placenta previa, intrauterine growth restriction, and perinatal mortality. Hemorrhage in the fundus of the uterus is reported to have poor prognosis compared with that in the lower uterine segment. Similarly, insufficient angiogenesis is associated with early pregnancy losses, and maternal serum alfa-fetoprotein (AFP) and $\beta$-human chorionic gonadotropin (hCG) are suggested to be used as markers of angiogenesis in the first trimester. Together with these markers, chronic inflammation of the decidua might also be the underlying cause of early pregnancy bleedings. ${ }^{6}$

Maternal age, vaginal bleeding, and gestational age at the time of the diagnosis are possible outcome factors in pregnancies complicated by a hematoma in the first trimester. Maternal age is considered an independent risk factor for adverse outcome because there is a strong correlation with chromosomal and structural fetal anomalies. The gestational age at diagnosis was related to spontaneous abortion. There is no correlation between vaginal bleeding and the adverse outcomes.

Intrauterine hemorrhages are commonly observed features on ultrasound examinations, especially, among patients with clinically evident bleeding in early pregnancy, and the incidence has been reported to be 4 to $22 \%$, depending on the patient population studied.

Subchorionic hematomas (SCHs) usually appear as hypoechoic or anechoic crescent-shaped areas on ultrasonography, a sonolucent fluid collection behind the fetal membranes or the placenta. The position of the hematoma relative to the placental site was described as subchorionic or retroplacental. The $\mathrm{SCH}$ is defined as being located between the chorion and the uterine wall, while the retroplacental hematoma is located behind the placenta. The location of the hematoma is marked as anterior, posterior, fundal, or cervical.

Although the exact etiology is uncertain, they are believed to result from partial detachment of the chorionic membranes from the uterine wall. Uterine malformations, history of recurrent pregnancy loss, and infections are the possible predisposing factors. The clinical significance of
$\mathrm{SCH}$ remains controversial. It is also not certain if these hemorrhages result in abortion. However, according to the results of a recent meta-analysis, the presence of $\mathrm{SCH}$ increases the risk of early or late pregnancy loss by twofold. It is suggested that the presence of $\mathrm{SCH}$ increases the risk of an adverse obstetric outcome, and fetal outcome is associated with the size of the hematoma, maternal age, and gestational age. ${ }^{6}$

Also a controversial issue is the association between the size of the hematoma and subsequent pregnancy complications. There are different classifications for the size of the hematoma. The hematoma can be classified as small, medium, or large according to the size relative to the GS. Another option is to measure the volume of the hematoma. Volumes are estimated by measuring the anteroposterior, longitudinal, and transverse diameters and multiplying these values by 0.523 (the ellipsoid formula). Because the hematoma may wrap around the GS, the ellipsoid formula would overestimate the size. ${ }^{7}$

To the best of the authors' knowledge, it is not the volume, but the position of hematoma that determines the prognosis. A subchorionic hemorrhage under the chorion laeve can reach large dimensions without any serious consequence; while if it is the insertion site of umbilical cord, a small volume can lead to abortion. Subchorionic hemorrhage near the cervix can clear spontaneously from the uterus; thus, prognosis is good. Fresh blood means immediate clearing, brown bleeding indicates previous hemorrhage. A hematoma near the fundus can increase the intrauterine volume significantly, causing the termination of pregnancy. ${ }^{8-10}$

Mäkikallio et a ${ }^{11}$ measured the pulsatility index (PI) of uterine, arcuate, spiral, umbilical, and chorionic arteries in first trimester pregnancies with vaginal bleeding or $\mathrm{SCHs}$. They found that vaginal bleeding with or without a $\mathrm{SCH}$ is associated with increased radial artery impedance at the 7th week of pregnancy. Persistence of the SCH does not affect uteroplacental and umbilicoplacental circulation.

The subchorionic hemorrhage is isoechogenic with the endometrium, and slow blood flow can be detected inside it. With abortion, the hematoma becomes hypoechoic then anechoic. If the hemorrhage does not cause an abortion, an increased risk for placental complications can be expected.

The risk for preeclampsia, intrauterine growth retardation, and cesarean section due to intrauterine asphyxia increases significantly. All these complications could probably be traced back to the same problem with placentation. The etiology can be mainly undetermined. ${ }^{12}$

In contrast to subchorionic hemorrhage of chorion laeve, the hemorrhage of chorion frondosum indicates a bad prognosis. Subchorionic hemorrhage is a fluidfilled cavity of variable size and echogenicity under the 
chorionic plate. Its incidence in a normal early pregnancy is 2 to $4 \%$. It increases the probability of abortion to twice the original incidence. In cases of imminent abortion, it is detected in every second pregnancy.

There is no association between the presence of clinical symptoms at the time of detection of the hematoma and poor perinatal outcome. Interestingly, a retroplacental position of the hematoma is significantly correlated with an increased risk for adverse maternal and neonatal complications.

The rate of cesarean section and vacuum extraction is significantly higher in patients with hematoma diagnosed in the first trimester of pregnancy. Pregnancy-induced hypertension and preeclampsia are more common in pregnant women with subchorionic or retroplacental hemetoma, and also the placental abruption. The rate of preterm delivery, intrauterine growth restriction, fetal distress, and neonatal intensive care unit admission increased significantly in patients with extravillous hematoma.

In cases of retroplacental hematomas, relative risk for:

- Cesarean delivery

- Hypertension

- Preeclampsia

- Placental abruption

- Placental separation abnormalities

- Preterm delivery

- Fetal growth restriction

- Fetal distress

There is no correlation between the size of the hematoma and the adverse perinatal outcome. ${ }^{12}$ The presence and the characteristic of an intrauterine hematoma during the first trimester may identify a population of patients at increased risk for adverse pregnancy outcome. Indeed, the ultimate goal of triaging patients into low-risk and high-risk populations is to more accurately target the available forms of surveillance and therapy.

In practice, it is advisable to classify every pregnancy with subchorional hemorrhage among the high-risk group. Repeated ultrasound imaging due to lack of therapeutic results is not advisable.

\section{YOLK SAC}

\section{Development of the Yolk Sac}

Between the 22nd and 28th postmenstrual days, the embryo contains only two layers - the embryonal ectoderm and the primary endoderm. The two layers form two cavities around the embryo - the amniotic cavity (ectoderm) and the primary yolk sac (endoderm). The secondary yolk sac evolves from the primary yolk sac by the 5th postmenstrual week (29-36 days). By this time, the primary yolk sac is absorbed and the secondary yolk sac has completely developed. The extraembryonic mesoderm which fills the cavity of the blastocyst is converted into a thin layer, covering both the amnion and the yolk sac, forming somato- and splanchnopleura. These two shields communicate only by the body-stalk.

The growth rate of the yolk sac is quicker than that of the amnion cavity. At the beginning of the 5th week, it is the first visible structure in the chorionic cavity. It is a round structure with an echogenic rim and a hypoechoic center. At this time, it is 3 to $4 \mathrm{~mm}$. The secondary yolk sac is the unambiguous evidence of intrauterine gravidity. Until there is a detectable yolk sac inside the uterine cavity, we should always take ectopic pregnancy into account.

Around 36 to 38 postmenstrual days, the embryo becomes visible between the amnion cavity and the yolk sac. It is only a 2 to $3 \mathrm{~mm}$ long, linear, hyperechoic structure. Even at this stage, we can detect the embryo's heart activity, although there is no detectable circulation in the yolk sac until the 6th week. By the 9th week, the yolk sac grows to a diameter of 5 to $6 \mathrm{~mm}$, but it begins to degenerate soon after and disappears by the 12 th week. ${ }^{13}$

\section{Structure of the Yolk Sac}

The yolk sac is made up of three layers: (i) The inner endoderm, (ii) the middle mesenchymal, and (iii) the external mesothelial layer. The inner endodermal layer contains 10 to $20 \mu \mathrm{m}$ wide columnar cells, with 0.5 to $1.0 \mu \mathrm{m}$ long cilia on the surface. Inside the cells, there are mitochondria, a highly developed Golgi apparatus, lysosomes, glycogen, and intracellular vacuoles. These cells are very similar to the liver cells due to their similar functions. The canalicular network of these cells also resembles that of the liver. The middle layer is the mesenchymal layer. It contains blood vessels, red blood cells, and macrophages. The vessels spring from the vitellointestinal duct. There is no basal membrane between the inner and the middle layer. The external layer is the mesothelial layer containing 5 to $10 \mu \mathrm{m}$ high cells with 2 to $5 \mu \mathrm{m}$ microvillous on the surface. The cells are full of intracellular vacuoles. After the 9th week, its structure changes. The microvillous and the inner cells structures dissolve, and the yolk sac begins to degenerate.

The yolk sac is the first anatomic structure identified within the GS. The yolk sac is a membranous sac attached to an embryo, formed by cells of the hypoblast adjacent to the embryonic disk. The yolk sac is situated on the ventral aspect of the embryo; it is lined by extraembryonic endoderm, outside of which is a layer of extraembryonic mesenchyme, derived from the mesoderm. Blood is conveyed to the wall of the yolk sac by the primitive aorta, and after circulating through a wide-meshed capillary plexus, is returned by the vitelline veins to the tubular 
heart of the embryo. This constitutes the vitelline circulation, and by means of it, nutritive material is absorbed from the yolk sac and conveyed to the embryo.

At the end of the 4th week, the yolk sac presents the appearance of a small pear-shaped opening (traditionally called the umbilical vesicle), into the digestive tube by a long narrow tube, the vitelline duct. It becomes visible at 5 weeks of gestation and normally degrades between 10 and 12 weeks. It is spherical in shape, with a welldefined echogenic periphery and a sonolucent center. Both the size and appearance of the yolk sac should be considered in early pregnancy. Yolk sac diameter correlates poorly with gestational age, so it is not used for gestational dating.

\section{Function of the Yolk Sac}

Due to its structure and position, the yolk sac plays an important role in nutrition transport. The following facts support this role:

- The wall and the cavity of yolk sac are in direct contact with the primitive midgut

- Its histological structure is very similar to the liver

- The composition of the celomic fluid is significantly different from the amniotic fluid. It contains proteins, creatinine, and hCG in a higher concentration ${ }^{14}$

- The yolk sac synthesizes numerous proteins, which are later produced by the liver including AFP, alfa1-antitrypsine, albumin, prealbumin, and transferrin. Till the 10th postmenstrual week, these factors are produced by the yolk sac and after that by the liver.

\section{Circulation of the Yolk Sac}

About the end of the 5th week, mesoderm cells located in visceral mesoderm of the wall of the yolk sac differentiate into blood cells and blood vessels. Centrally located cells then give rise to primitive blood cells, while those on the periphery flatten and form endothelial cells lining blood islands. Blood islands approach each other rapidly by sprouting of endothelial cells and after fusion, give rise to small vessels. At the same time, blood cells and capillaries develop in the extraembryonic mesoderm of the villous stems and connecting stalk. By continuous budding, extraembryonic vessels establish contact with each other inside the embryo. Intraembryonic blood vessels, including the heart tube, are established in exactly the same manner as extraembryonic vessels. The rhythmic contractions of the heart pump the primitive blood from connecting stalk toward cranial portion of the embryo. Meanwhile, the intraembryonic blood vessels protrude into the chorion through body stalk, and form capillary loops at the axis of villi giving rise to the placental circulation. The intraembryonic circulation precedes blood flow in the intervillous space. Normal placental circulation starts only after the end of the organogenesis around the 13th week, which confirms significant role of the yolk sac in nutritive and transport functions.

Kurjak and Kupesic ${ }^{15,16}$ examined the circulation of yolk sac and vitelline duct in early pregnancy. Before the 6th week, there is no detectable circulation in the bodystalk or the yolk sac by ultrasound Doppler examination. Between 6 and 12 weeks, there is a noncontinuous, low-velocity waveform with absence of diastolic flow (Figs 4A and B).

Overall visualization rate for yolk sac vessels was $80 \%$. The highest visualization rates were obtained in the 7th and 8 th weeks of gestation reaching values of $90 \%$. In the same period, the visualization rates of the vitelline duct arteries were 87 and $91 \%$. A characteristic waveform profile included low velocity $(5.8 \pm 1.7 \mathrm{~cm} / \mathrm{s})$ and absence of diastolic flow, which was obtained from all examined yolk sacs. The PI showed the mean value of $3.24 \pm 0.94$. Vitelline vessels showed similar peak systolic velocity $(5.4 \pm 1.8 \mathrm{~cm} / \mathrm{s})$ and PI values $(3.14 \pm 0.91) .{ }^{17}$
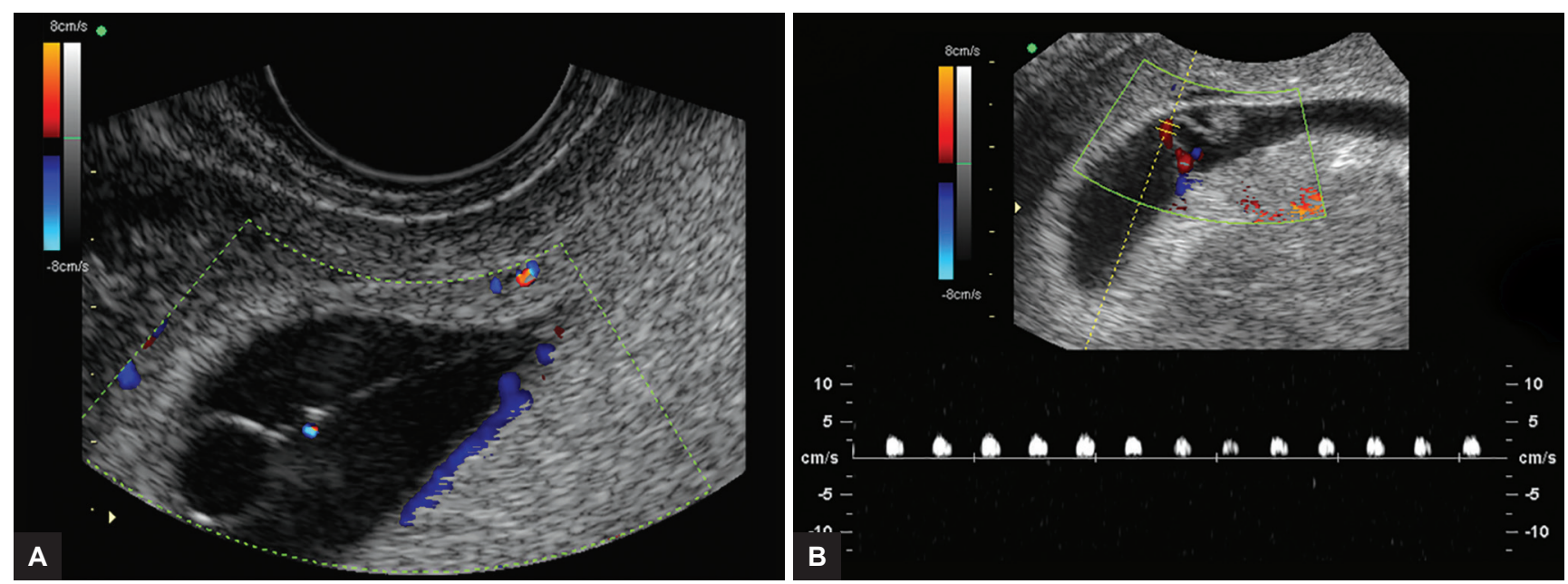

Figs 4A and B: The circulation of the yolk sac 


\section{Normal Yolk Sac}

At the 4th week of embryologic development, the wall of the yolk sac consists of three thin cellular layers. The outermost layer is the ectoderm, which faces the exocoelomic cavity. The ectodermal layer is in fact a distinct layer of flattened cells. However, the innermost layer facing the yolk sac cavity is the endodermal epithelium, which is composed of a single layer of cuboidal epithelial cells. Between these two layers, there is the mesodermal layer, which consists of blood island formations in which hematopoietic stem cells can be identified throughout a primitive capillary network.

A yolk sac can be detected easily by transvaginal sonography when the mean GS diameter is 5 to $6 \mathrm{~mm}$. It is generally accepted that the yolk sac should be observed when a GS measures greater than $8 \mathrm{~mm}$. The yolk sac is connected to the embryo by the vitelline duct. Normally, the yolk sac appears as a circular structure with an anechoic center surrounded by a uniform well-defined echogenic wall. Usually, the inner diameter of a yolk sac measures 3 to $5 \mathrm{~mm}$. In fact, the yolk sac size progressively increases from the beginning of the 5 th gestational week to the end of the 10th gestational week. Afterward, the yolk sac size decreases gradually. Abnormal yolk sac diameters are generally reported to be associated with subsequent pregnancy failure.

\section{Abnormalities of Yolk Sac Development}

It is evident from the formation and function of yolk sac that any deviation in these complicated processes could disturb the development of embryo. It plays an important role in the nutritive, metabolic, and hematopoietic processes of the first trimester.

Any abnormality in the shape, size, structure or circulation of the yolk sac indicates a major abnormality in development.

The absence of yolk sac is the first sonographic indicator of an early maldevelopment. It is a very important ultrasound finding of a blighted ovum. In this case, we should distinguish between intrauterine and ectopic pregnancy. On transvaginal sonography, absence of the yolk sac in the presence of an embryo is always abnormal and, in general, is associated with subsequent embryonic death. ${ }^{18}$

Abnormal yolk sac size is also an indicator of maldevelopment. ${ }^{19}$ Established that for a GS diameter of less than $10 \mathrm{~mm}$, the yolk sac diameter should be less than $4 \mathrm{~mm}$. A large yolk sac can indicate poor pregnancy outcome. In the surviving embryos, the probabilities for chromosomal aberrations increase (Fig. 5). Although there is no clearly identified consensus, most authors accept either 5 or $6 \mathrm{~mm}$ as the upper limit for the size of a normal

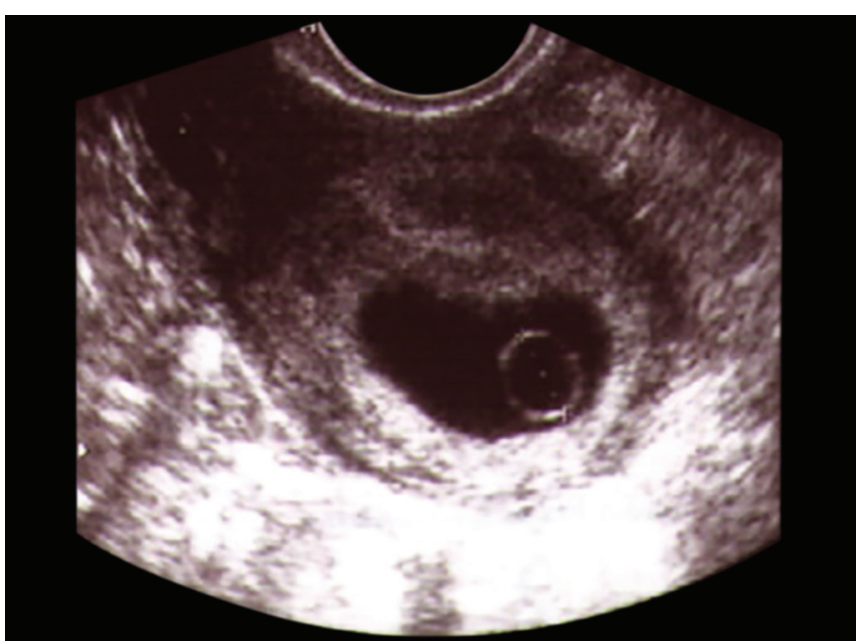

Fig. 5: Large yolk sac

yolk sac in pregnancies with a gestational age from the 5 th to the 10th weeks. Generally, it has been suggested that an abnormally large yolk sac may indicate a poor obstetric outcome; therefore, close follow-up with sonography is recommended for these pregnancy outcomes. ${ }^{18}$

Small yolk sac can also predict poor pregnancy outcome. Green and Hobbins ${ }^{20}$ reported a similar outcome with diameter less than $2 \mathrm{~mm}$. Küçük et $\mathrm{al}^{21}$ found that the yolk sac diameter out of two standard deviations of the mean for gestational age allowed prediction of an abnormal pregnancy outcome with sensitivity of $65 \%$, a specificity of $97 \%$, a positive predictive value of $71 \%$, and a negative predictive value of $95 \%$. An abnormal yolk sac shape allowed prediction of an abnormal pregnancy outcome with a sensitivity of $29 \%$, a specificity of $95 \%$, a positive predictive value of $47 \%$, and a negative predictive value of $90.5 \%$.

It has been claimed that a very small yolk sac may be a normal finding during early periods of normal embryologic development. On the contrary, a much earlier published study, with certain limitations, suggests that a yolk sac diameter of $2 \mathrm{~mm}$ or less may be associated with an adverse outcome in pregnancies with a gestational age of 8 to 12 weeks. ${ }^{18}$

It is well known that the yolk sac size begins to decrease during the late weeks of the first trimester. This process is why gestational age should be taken into account when the size of the yolk sac is assessed. However, it would be prudent to perform serial sonographic examinations within a short period whenever a smaller-than-expected yolk sac has been visualized. ${ }^{22}$

Changes in echogenicity can also be the same predicting factors (Fig. 6). Presence of hyperechogenic yolk sac is highly associated with chromosomal aneuploidy between 9th and 11th gestational weeks. ${ }^{23}$

A calcified yolk sac may appear as an echogenic ring on sonography. It has not been reported to be associated 


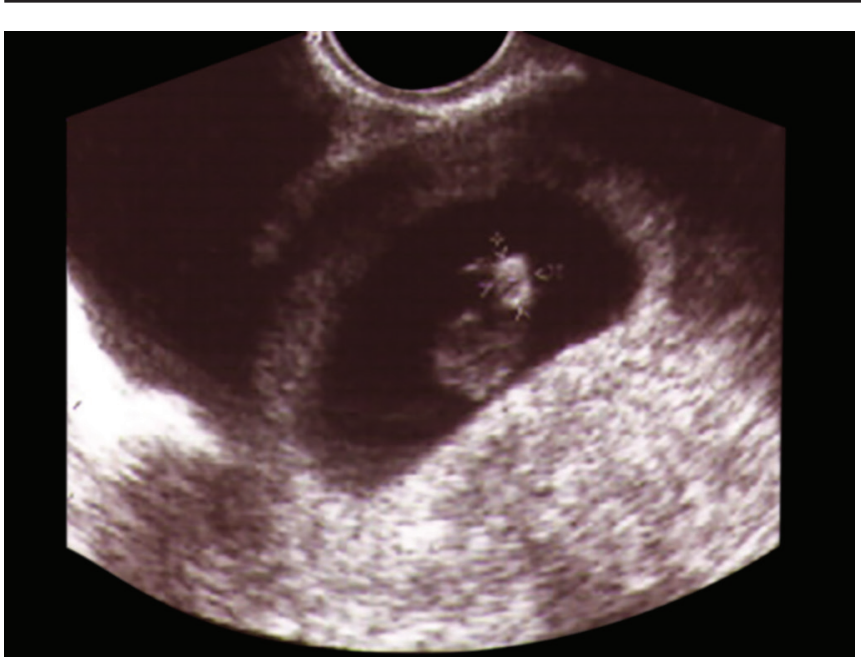

Fig. 6: Small hyperechoic yolk sac

with a live embryo before the 12th week of gestation. Indeed, a calcified yolk sac would be observed only with a dead embryo because the yolk sac would undergo calcification within a few days after embryonic death has occurred. ${ }^{18}$

An echogenic yolk sac is different from a calcified yolk sac in that echogenicity may represent various types of materials other than calcium. In the related literature, it has been anecdotally emphasized that an echogenic yolk sac does not predict an embryonic anomaly or death. In fact, large-scale prospective studies are anticipated to clarify the prognostic importance of echogenic yolk sacs.

When the 10th or 11th week of gestation is completed, the yolk sac begins to shrink rapidly and eventually disappears. However, sometimes the yolk sac can persist between the amnion and the chorion even after 12 weeks' gestation. The persistent yolk sac usually stands close to the site where the umbilical cord enters the placenta. The clinical importance of a persistent yolk sac is unknown. ${ }^{18}$

Kupesic and Kurjak $^{17}$ demonstrated the characteristics of yolk sac circulation. They found a noncontinuous low velocity waveform with absent diastolic flow. They also detected three different types of abnormal circulation signals in patients with missed abortions: (i) Irregular blood flow, (ii) permanent diastolic flow, and (iii) venous blood flow.

Mäkikallio et $\mathrm{al}^{11}$ examined the Doppler parameters of uterine, spiral, intraplacental, chorionic, umbilical, and yolk sac hemodynamics in early pregnancies. They found that in patient, who later had preeclampsia at week 8 , the maternal intraplacental resistance index (RI) was higher. A week later, the yolk sac RI and umbilical artery mean velocity were lower. In late first trimester, increased velocities and RI were observed in chorionic arteries. No difference in uterine, spiral artery hemodynamics, and in umbilical artery PI was observed. ${ }^{24}$
Table 2: Normal and abnormal yolk sac characteristics

\begin{tabular}{lll}
\hline & Normal findings & Abnormal findings \\
\cline { 2 - 3 } Characteristic & $\begin{array}{l}\text { Echogenic rim, } \\
\text { hypoechoic center }\end{array}$ & Hyperechoic \\
\hline Shape & Round & Distorted \\
Size & $3-4 \mathrm{~mm}$ in the & $<2 \mathrm{~mm}$, or $>6 \mathrm{~mm}$ \\
& 5th week & \\
& $5-6 \mathrm{~mm}$ in the & \\
& 10th week & \\
Doppler & Noncontinuous, & Irregular blood flow, \\
& absence of diastolic & $\begin{array}{l}\text { permanent diastolic flow, } \\
\text { venous blood flow }\end{array}$ \\
& flow &
\end{tabular}

In diamniotic twin pregnancies, the number of the embryos equals the number of yolk sacs. In monochorionic monoamniotic twin pregnancies, a single yolk sac may be a normal finding. But, in cases of single yolk sac, they are highly associated with conjoined twins (Table 2). ${ }^{19}$

\section{REFERENCES}

1. Özkaya E, Altay M, Gelişen O. Significance of subchorionic haemorrhage and pregnancy outcome in threatened miscarriage to predict miscarriage, pre-term labour and intrauterine growth restriction. J Obstet Gynaecol 2011 Mar;31(3):210-212.

2. Doubilet $P$, Benson CB. First do no harm ... to early pregnancies. J Ultrasound Med 2010 May;29(5):685-689.

3. Dighe M, Cuevas C, Moshiri M, Dubinsky T, Dogra VS. Sonography in first trimester bleeding. J Clin Ultrasound 2008 Jul-Aug;36(6):352-366.

4. Kurjak, A.; Chervenak, FA. Donald school textbook of ultrasound in obstetrics and gynecology. 3rd ed. Berlin: JP Medical Ltd; 2011. p. 1022.

5. Tongsong T, Wanapirak C, Srisomboon J, Sirichotiyakul S, Polsrisuthikul T, Pongsatha S. Transvaginal ultrasound in threatened abortions with empty gestational sacs. Int J Gynaecol Obstet 1994 Sep;46(3):297-301.

6. Kutluer G, Çiçek NM, Moraloğlu Ö, Ertargın P, Sarıkaya E, Artar I, Erdem O. Low VEGF expression in conceptus material and maternal serum AFP and $\beta$-hCG levels as indicators of defective angiogenesis in first-trimester miscarriages. J Turk Ger Gynecol Assoc 2012 Jun;13(2):111-117.

7. Leite J, Ross P, Rossi A, Jeanty P. Prognosis of very large firsttrimester hematomas. J Ultrasound Med 2006 Nov;25(11): 1441-1445.

8. Kurjak A, Schulman H, Zudenigo D, Kupesic S, Kos M, Goldenberg M. Subchorionic hematomas in early pregnancy: clinical outcome and blood flow patterns. J Matern Fetal Med 1996 Jan-Feb;5(1):41-44.

9. Ball RH, Ade CM, Schoenborn JA, Crane JP. The clinical significance of ultrasonographically detected subchorionic hemorrhages. Am J Obstet Gynecol 1996 Mar;174(3):996-1002.

10. Pearlstone M, Baxi L. Subchorionic hematoma: a review. Obstet Gynecol Surv 1993 Feb;48(2):65-68.

11. Mäkikallio K, Tekay A, Jouppila P. Effects of bleeding on uteroplacental, umbilicoplacental and yolk-sac hemodynamics in early pregnancy. Ultrasound Obstet Gynecol 2001 Oct;18(4):352-356.

12. Nagy S, Bush M, Stone J, Lapinski RH, Gardó S. Clinical significance of subchorionic and retroplacental hematomas 
detected in the first trimester of pregnancy. Obstet Gynecol 2003 Jul;102(1):94-100.

13. Jauniaux E, Jurkovic D, Henriet $Y$, Rodesch F, Hustin J. Development of the secondary yolk sac: correlation of sonographic and anatomic features. Hum Reprod 1991 Sep;6(8): 1160-1166.

14. Jauniaux E, Jurkovic D, Gulbis B. Biochemical composition of exocoelomic fluid in early human pregnancy. Obstet Gynecol 1991 Dec;78(6):1124-1128.

15. Kurjak A, Kupesic S, Kostovic L. Vascularization of yolk sac and vitelline duct in normal pregnancies studied by transvaginal color and pulsed Doppler. J Perinat Med 1994;22(5):433-40.

16. Kurjak A, Kupesic S. Color Doppler in obstetrics, gynecology and infertility. London: Parthenon Publishing Group; 1999.

17. Kupesic S, Kurjak A. Volume and vascularity of the yolk sac assessed by three-dimensional and power Doppler ultrasound. Early Pregnancy 2001 Jan;5(1):40-41.

18. Tan S, Ipek A, Pektas MK, Arifoğlu M, Teber MA, Karaoğlanoğlu M. Irregular yolk sac shape: is it really associated with an increased risk of spontaneous abortion? J Ultrasound Med 2011 Jan;30(1):31-36.
19. Levi CS, Lyons EA, Dashefsky SM, Lindsay DJ, Holt SC. Yolk sac number, size and morphologic features in monochorionicmonoamniotic twin pregnancy. Can Assoc Radiol J 1996 Apr;47(2):98-100.

20. Green JJ, Hobbins JC. Abdominal ultrasound examination of the first trimester fetus. Am J Obstet Gynecol 1988 Jul;159(1):165-175.

21. Küçük T, Duru NK, Yenen MC, Dede M, Ergün A, Başer I. Yolk sac size and shape as predictors of poor pregnancy outcome. J Perinat Med 1999 Sep;27(4):316-320.

22. Berdahl DM, Blaine J, Van Voorhis B, Dokras A. Detection of enlarged yolk sac on early ultrasound is associated with adverse pregnancy outcomes. Fertil Steril 2010 Sep;94(4):1535-1537.

23. Szabó J, Gellén J, Szemere G, Faragó M. Significance of hyperechogenic yolk sac in the first-trimester screening for chromosome aneuploidy. Orv Hetil 1996 Oct;137(42): 2313-2315.

24. Mäkikallio K, Jouppila P, Tekay A. First trimester uterine, placental and yolk sac haemodynamics in pre-eclampsia and preterm labour. Hum Reprod 2004 Mar;19(3):729-733. 